\title{
Estimation of Rainfall Induced Soil Erosion An Experimental Study
}

\author{
K.P.P. Pathirana, T.M. Asmer, S.M. Fahim and A.L.A. Hyther
}

\begin{abstract}
Soil erosion is a serious global problem with significant financial and environmental consequences and more studies are needed to understand the physical mechanism of erosion and also to quantify erosion rates. Rainfall induced soil erosion is a two-phase process consisting of the detachment of individual soil grains from soil mass and their transport by running water. Although several methods are available for estimating rainfall induced soil erosion, the applicability of most of these methods are very limited. This paper presents a detailed study carried out using a laboratory set-up consisting of an artificial rainfall simulator to quantify rainfall induced soil erosion. Based on a series of laboratory experiments carried out with different soils and rainfall intensities, two functional relationships were developed to quantify erosion rates on sandy soils and clayey soils separately These equations were able to predict erosion rates to a reasonable accuracy and also easy to use in actual situations. However, further tests are recommended with more soil samples and widening the range of other test parameters to verify the equations and to make it applicable over a wide range of actual situations.
\end{abstract}

Keywords: Rainfall induced soil erosion, raindrop size distribution, rainfall intensity

\section{Introduction}

Soil erosion is a process which involves the detachment and removal of soil particles from the land surface. It is a natural physical phenomenon responsible in shaping the present form of earth's surface with hills and valleys. Soil erosion may be important for soil formation however, with respect to soil degradation, most concerns about erosion are related to accelerated erosion, where the natural rate has been significantly increased mostly by human activity. With the increase in population, the pressure on land increase, and leads to its overexploitation, and subsequently, its degradation. It is well known that the soil erosion is a widespread problem throughout the world with significant financial and environmental consequences. Soil erosion is particularly important on agricultural lands. By removing the most fertile topsoil, erosion reduces soil productivity. Though fertilizer consumption is increased in order to maintain yield on the remaining shallow soil layer, may finally lead to land abandonment, resulting an irreversible loss of natural farmland (Dimoyiannis et al., 2001). In addition, the problems of sedimentation on riverbeds and drainage networks, which reduce their capacity, increasing flooding risk, block irrigation canals and shorten the design life of reservoirs. Sediment is also act as a primary source of pollution and, through the chemicals absorbed to it, can increase the level of nitrogen and phosphorous in water bodies. Therefore, it is very important to investigate the mechanism of soil erosion with reliable predictive capabilities on erosion rates.

The principal agents responsible for soil crosion are wind and water. Wind erosion generally prevails in arid and semi-arid areas where rainfall is low and the day temperatures are high Water erosion occurs in areas where rainfall is relatively high with a heavy runoff on sloping lands and which is the area of present study.

\section{Rainfall Induced Soil Erosion}

Soil erosion is a two-phase process consisting of the detachment of individual soil particles from

Eng.(Dr.) K.P.P. Pathirana, B.Sc. Eng. (Hons.) (Peradeniya) M.Eng., Ph.D., C.Eng., FIE(Sri Lanka), MICE(London), Senior Lecturer, Department of Civil Engincering, University of Peradeniya.

T.M. Asiner, B.Sc. Eng. (Hons), Department of Civil Engineering, University of Peradeniya.

S.M. Fahim, B.Sc. Eng. (Hons), Department of Civil Engineering, University of Peradeniya.

A.L.A. Hyter, B.Sc. Eng. (Hons), Departuent of Civil Engincering, University of Peradeniva. 
the soil mass and their transport by erosive agent such as running water (Van Dijk et al., 2003). The impact of raindrops on the soil surface can break down soil aggregates and disperse the aggregate material. Lighter materials such as, very fine sand, silt, clay and organic matter can be easily removed by the raindrop splash and runoff water, but require much greater raindrop energy or runoff amounts to move the larger sand and gravel particles. When sufficient energy is no longer available to transport the particles a third phase, deposition occurs.

There are many factors influencing the soil erosion such as, rainfall intensity, runoff, grain size, erodability of soil, land slope, nature of the plant cover etc. Therefore, analytical approach to investigate the problem of rainfall induced soil erosion is generally impossible, and hence, most of the studies are mainly based on laboratory and field investigations.

Universal Soil Loss Equation (USLE) has been identified as one of the well established methods available for estimating erosion rates at present. USLE was originally developed to estimate sheet and rill erosion losses from cultivated fields in the United States and it is now also applied to regions outside the US (Morgen, 1995). One of the major difficulties in using USLE is to find appropriate values for many different parameters used in this method and the guidelines available for finding these parameters are also limited only to the regions in the US. In addition, there are few other studies reported in literature attempting to suggest much improved methods for predicting erosion rates, but the applicability of most of these methods are limited.

The present study describes an extensive laboratory experiments carried out using an artificial rain simulator to investigate rainfall induced soil erosion mainly to develop a functional relationship to quantify erosion rates.

\section{Experimental Details}

\subsection{Preliminary Tests}

Prior to design and fabrication of a rainfall simulator for the proposed experimental studies, it is necessary to know the characteristics of natural rainfall. The most important characteristics of natural rainfall that need to be closely simulated for soil erosion studies are raindrop size distribution, raindrop impact velocity and appropriate rainfall intensities. Out of these, raindrop impact velocity is difficult to measure and control in laboratory experiments. In addition, it is also much more challenging to simulate both raindrop impact velocity and the correct drop size distribution simultaneously in a laboratory set-up. Hence, raindrop impact velocity was not considered in the proposed rain simulator.

Several tests were carried out to obtain the drop size distribution of natural rain using flour pellet method described in Hudson (1963). A tray filled with flour was exposed to rain just for very short time and the number of pellets formed on the flour surface with different diameters was counted (Figure 1).

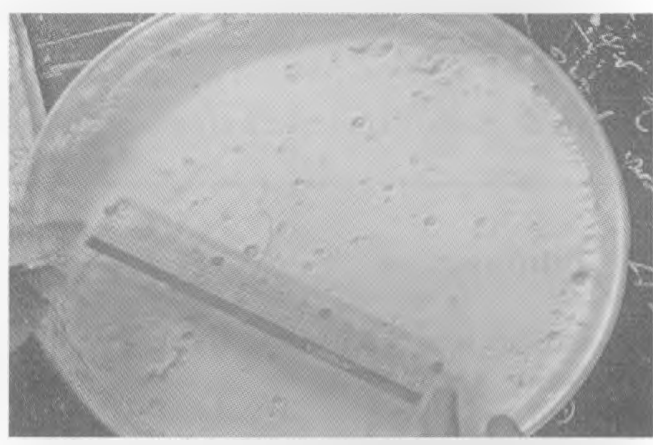

Figure 1 - Pellets formed on flour during field test

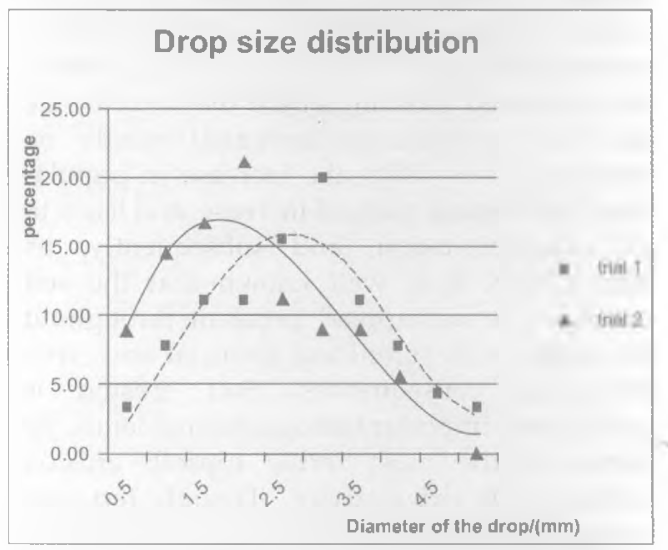

Figure 2 - A typical test result of raindrop size distribution.

Although a simple method was used here to obtain the raindrop size distribution, the distributions obtained were reasonable for the proposed study. The number of very small drops may not be accurately estimated here, however, very small raindrop are not of much interest to detachment of soil grains. Figure 2 
shows typical test results of raindrop size distributions.

\subsection{Rainfall Simulator}

A laboratory scale rainfall simulator was designed to produce the drop size distribution as similar to one obtained for natural rain and also to get a wide range of rainfall intensities. The arrangement consists of a set of perforated PVC pipes and four spray nozzles mounted on a movable frame as shown in Figure 3. The nozzles were positioned in such a way that spray pattern of one nozzle overlaps with the others. The frame is also movable back and forth to a span of about $10 \mathrm{~cm}$ using a viper motor. The purpose of this set-up movable was to obtain the correct drop size distribution of the rain simulated in the laboratory as similar to a natural rain. The perforated pipes produce relatively larger water drops whereas; the spray nozzles emit smaller drop sizes so that the combination of both would be able to generate a raindrop size distribution reasonably closer to a natural rain. The height of the simulator above the ground level was determined after carrying out several preliminary test runs.

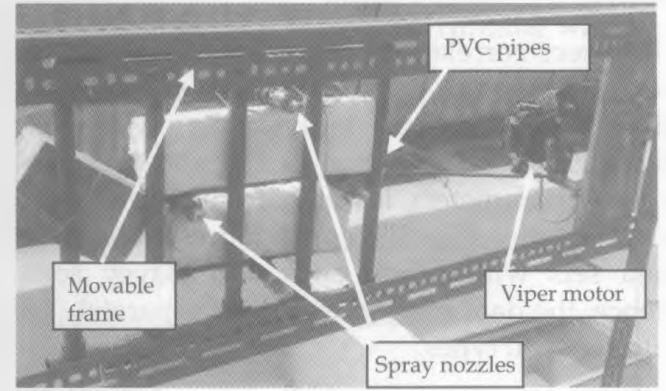

Figure 3 - Rainfall simulator

Figure 4 shows a comparison of drop size distribution simulated by the rainfall simulator with that of an actual rain. It can be noted that the larger raindrops were reasonably simulated in the rain simulator compared to smaller raindrops. This is acceptable as large rain dropsare more important for inducing soil erosion compared to smaller ones.

Uniformity of rainfall generated by the rain simulator was also tested by placing a set of containers kept in a grid pattern and measuring the rainfall volumes collected over a range of intensities and durations. Christiansen Uniformity Coefficient was used to compare the uniformity of rainfall generated by the rain simulator.

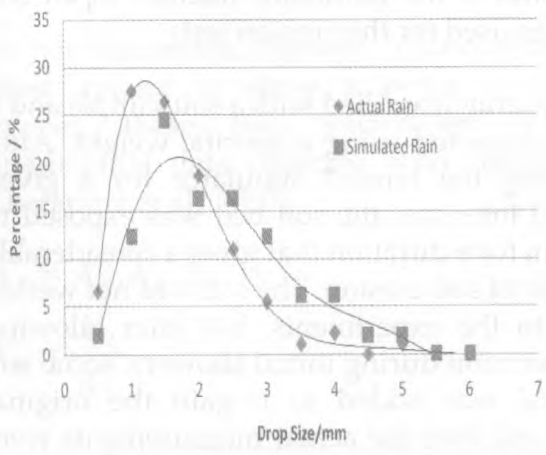

Figure 4 - A comparison of drop size distribution produced by the rain simulator with an actual rain.

\subsection{Experimental Procedure}

The soil tray with a size of $600 \times 600 \times 100 \mathrm{~mm}$ was used for the tests. It consists of $70 \mathrm{~mm}$ thick soil sample placed on a layer of $20-30 \mathrm{~mm}$ thick gavel layer which is to facilitate drainage through its larger pours and then, through a wire mesh fixed to the down-slope end of the soil tray. Both side of the soil bed was covered with wooden boards to prevent eroded soil to splash out of the tray. The complete experimental set-up used for erosion tests is shown in Figure 5.
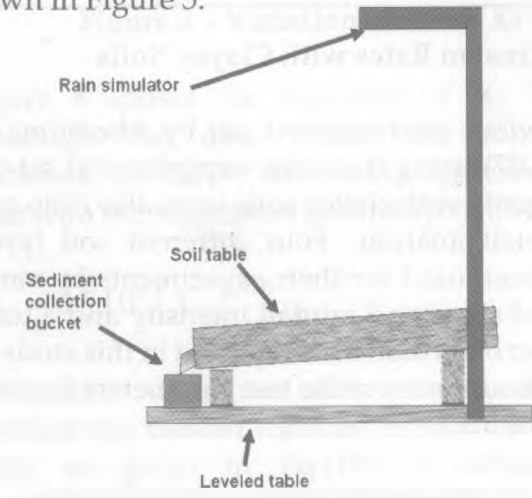

Figure 5 - Experimental set-up

Table 3.1: Texture of soil samples

\begin{tabular}{|c|c|c|c|c|}
\hline \multirow{2}{*}{ Sample } & \multicolumn{5}{|c|}{ Percentages } \\
\cline { 2 - 5 } & Clay & Silt & Sand & Gravel \\
\hline A & 0 & 12 & 76 & $\mathbf{1 2}$ \\
\hline B & 0 & 10 & 82 & 8 \\
\hline C & 2 & 31 & 60 & 7 \\
\hline D & 0 & 15 & 85 & 0 \\
\hline
\end{tabular}

The soil samples were classified after carrying out several laboratory tests, such as hydrometer test and sieve analysis. Four different soil types were used for the tests and soil textures of these samples are given in Table 3.1. It can be noted 
that sand is the dominant fraction in all soil samples used for the erosion tests.

The soil tray was filled with a soil sample and it was compacted using a specific weight. After adjusting the rainfall simulator for a given rainfall intensity, the soil bed was exposed to the rain for a duration that gives a considerable amount of soil erosion. The soil was not wetted prior to the experiments, but after allowing some erosion during initial showers, some soil material was added to re-gain the original level, and then the actual measurements were started. The eroded material was collected to a bucket placed at the downstream end of the soil bed. The above procedure was repeated for four different bed slopes and four rainfall intensities. With the four different soil samples, altogether 64 erosion tests were conducted. The range of test parameters used in this study is summarized in Table 3.2.

Table 3.2 - Summary of experimental data

\begin{tabular}{|l|c|}
\hline \multicolumn{1}{|c|}{ Parameter } & Range \\
\hline Soil bed slope & $14^{0}-32^{\circ}$ \\
Rainfall intensity $(\mathrm{mm} / \mathrm{hrs})$ & $70-175$ \\
Mean grain size $(\mathrm{mm})$ & $0.4-0.8$ \\
Mean raindrop size $(\mathrm{mm})$ & $0.8-1.5$ \\
\hline
\end{tabular}

\subsection{Erosion Rates with Clayey Soils}

A previous study carried out by Aberathna et al., (2007) using the same experimental set-up but mainly with clayey soils were also collected for detail analysis. Four different soil types have been used for their experiments by varying bed slope and rainfall intensity and a total number of erosion tests reported in this study is 80. The summary of the test parameters is given in Table 3.3 .

Table 3.3 - Summary of experimental data (Aberathne et al., 2007)

\begin{tabular}{|l|c|}
\hline \multicolumn{1}{|c|}{ Parameter } & Range \\
\hline Soil bed slope & $8^{0}-22^{0}$ \\
Rainfall intensity $(\mathrm{mm} / \mathrm{hrs})$ & $50-300$ \\
Mean grain size $(\mathrm{mm})$ & $0.11-0.5$ \\
Mean raindrop size $(\mathrm{mm})$ & $1.0-2.5$ \\
Clay content & $5-31$ \\
\hline
\end{tabular}

\section{Data Analysis}

The soil erosion rate $(q)$ depends on many parameters such as, rainfall intensity $(I)$, impact velocity of raindrops $(V)$, mean diameter of soil grains $(d s)$, mean diameter of raindrops $(d r)$, dry density of soil $(\rho)$, bed slope (S), acceleration of gravity (g), soil characteristics $(K)$, support factor to represent any erosion control measures $\left(P_{0}\right)$. These parameters can be written to an equation in a dimensional form;

$f\left(I, V, d_{s}, d_{r}, \rho, S, g, K, P_{0}, q\right)=0$

The parameter $P_{0}$ can be removed from the list as no erosion control measures were adopted in all the test runs. The impact velocity of raindrop was also not taken into account for the analysis as it is difficult to measure and control during the experiments. In addition, clay content of soil (C) was taken as the representative parameter of soil characteristics $(K)$. Hence, the Eq. (1) simplifies to;

$f\left(I, d_{s}, d_{r}, \rho, S, g, C, q\right)=0$

Using the Buckingham $\Pi$ - theorem, the following non-dimensional groups were formed.

$X_{1}=S ; X_{2}=\frac{q}{I \rho} ; X_{3}=\frac{d_{r}}{d_{s}} ; X_{4}=\frac{g d_{s}}{I^{2}} ;$

$X_{5}=C$

Where, $S=\tan (\theta)$ and $\theta=$ soil bed slope.

No direct correlations were found among the non-dimensional groups formed above when taking these groups individually. Moreover, even with a combination of the above dimensionless parameters, it was not possible to find a single relationship that could represent both the data sets with sandy soils and clayey soils. Hence, the data analyse was done separately for different soil types.

\subsection{Data Analysis for Sandy Soils}

It is clear that the non-dimensional group $X_{5}$ can be omitted in the analysis of experimental data for sandy soils. A new group, $X_{6}$ formed by combining $X_{1}, X_{3}$ and $X_{4}$ as given in Eq.(4), appear to have a reasonable correlation with $X_{2}$ for erosion rates obtained with sandy soils.

$X_{6}=X_{1}^{3} \cdot X_{3}^{2} \cdot X_{4}$

The variation of $X_{2}$ Vs $X_{6}$ is shown in Figure 6. It can be seen that a good correlation is obtained between $X_{2}$ and $X_{6}$ (Eq.5), despite some scatter of data points. This could be partly attributed to experimental errors. One of the difficulties faced during the experiments was to apply the same degree of compaction to the soil samples when preparing the soil bed prior to 
erosion tests which could have influenced the final results.

$X_{2}=10^{-7}\left(X_{0}\right)^{052}$

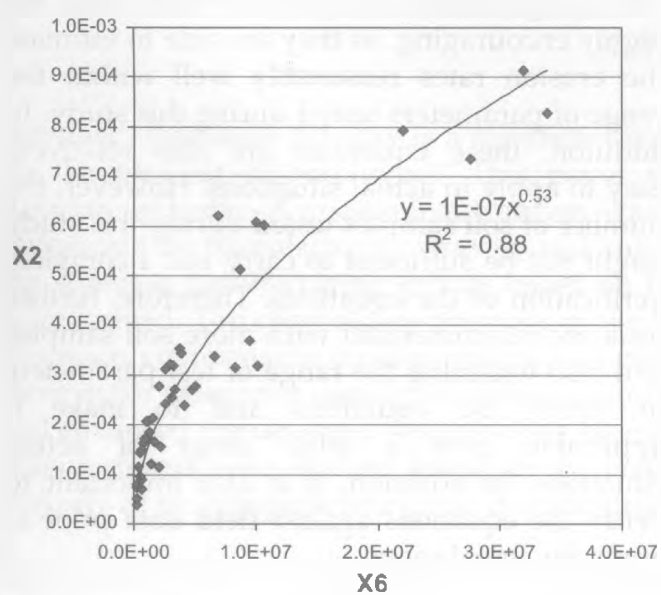

Figure 6 - Variation of $X_{2}$ Vs $X_{6}$

Failure to generate the correct number of smaller size raindrops in the laboratory rain. simulator (see Section 3.2) might have also been partly influenced the erosion rates, though they are unable to initiate erosion by detaching grains, but due to increase in discharge this might support the transport of detached soil particles down the slope with running water.

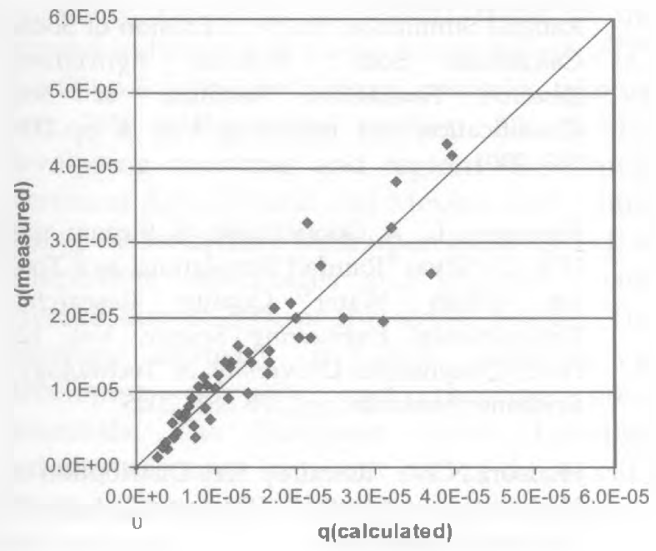

Figure 7 - Comparison between measured and computed erosion rates on sandy soils

Eq.(6) can be used to estimate the rainfall induced erosion on sandy soils. A comparison between the measured and computed erosion rates is illustrated in Figure 7.

$$
q=10^{-7}\left[S^{3} \cdot\left[\frac{d_{r}}{I}\right]^{2} \cdot \frac{g}{d_{s}}\right]^{0.53}(I \rho)
$$

\subsection{Data Analysis for Clayey Soils}

The non-dimensional groups, $X_{7}$ and $X_{8}$ derived below appear to show a better correlation between these two parameters for the experimental data collected with clayey soils (Aberathna et al., 2007).

$$
\begin{aligned}
& X_{7}=X_{2} \cdot X_{3} \cdot X_{4} \\
& X_{8}=\frac{X_{4}}{X_{1}^{2}}
\end{aligned}
$$

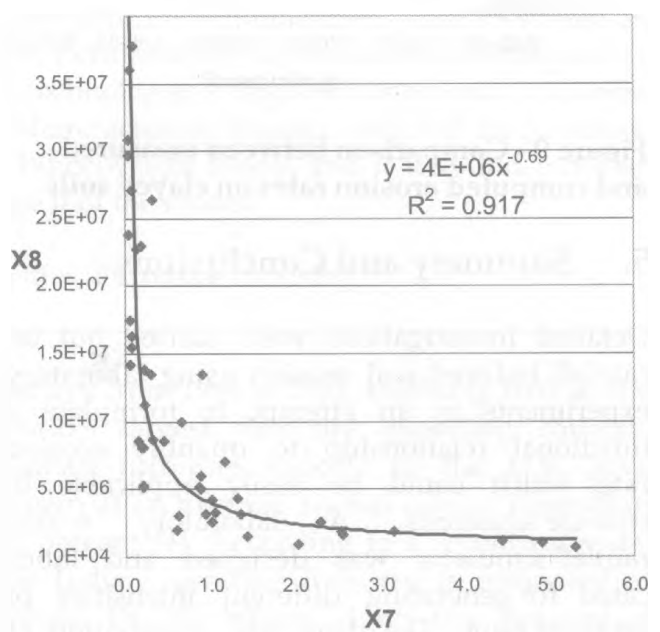

Figure 8 - Variation of $X_{8}$ Vs $X_{7}$

Figure 8 shows the variation of $X_{7} V_{5} X_{8}$. Although the data points are somewhat scattered, the Eq.(9) reasonably represents the data with the regression coefficient of 0.92 .

$X_{8}=4 * 10^{6}\left(X_{7}\right)^{-0.69}$

This allows us to use a separate equation to estimate the rainfall induced erosion on clayey soils, as given in Eq.(10). A comparison between the measured and computed erosion rates is shown in Figure 9.

$q=3.74 * 10^{9}\left[\left.\frac{S^{2} I^{2}}{g d_{s}}\right|^{1.45}\left[\frac{I \rho d_{i}}{d_{r} C}\right]\right.$

The deviation of results from the actual data could have been mainly due to the factors that are difficult to control during the experiments such as, initial moisture content and degree of compaction of soil layers etc.

Despite these shortcomings however, the proposed equations are able to reasonably predict the rainfall induced soil erosion rates. 


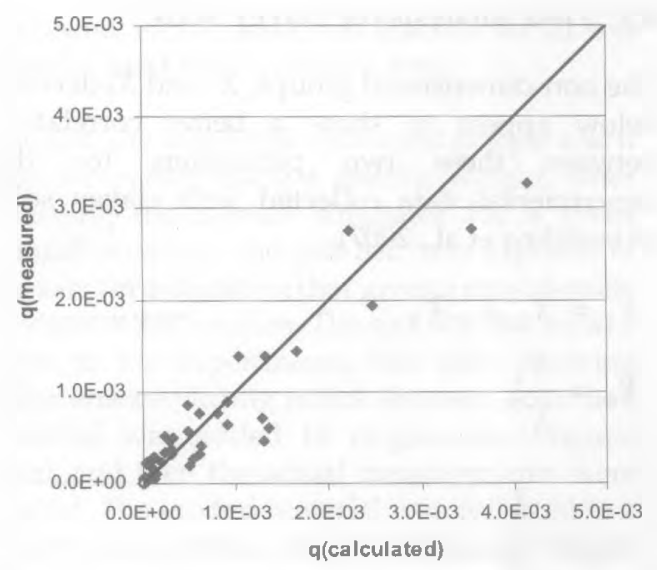

Figure 9 - Comparison between measured and computed erosion rates on clayey soils

\section{Summary and Conclusions}

Detailed investigations were carried out on rainfall induced soil erosion using laboratory expcriments in an attempt to formulate a functional relationship to quantify erosion rates which could be easily applicable in real-life situations. A laboratory scaled rainfall simulator was designed and fabricated for generating different intensities of artificial rains. The drop size distribution of the rainfall simulated is found to be reasonably comparable to natural rainfall. Although impact velocity of raindrops is an important parameter for rainfall induced soil erosion, it is very difficult to measure and control in laboratory tests while meeting the corresponding drop size distribution and rainfall intensity simultaneously. Hence, the raindrop impact velocity was not considered in the present study. The crosion tests were carried out using four different soil samples with sand being the dominant fraction. Each sample was tested for four rainfall intensities by placing the soil bed to four different bed slopes. The erosion rates carried out with clayey soils using the same laboratory set-up by Aberathna et al, (2007) were also collected.

Using dimensional analysis on the factors governing the rainfall induced soil erosion, two different functional relationships were developed for estimating the erosion rates on sandy soils and clayey soils separately. It is also noted that there were some deviations of the computed erosion rates from the measured values and some of which may be attributed to experimental errors and also to the factors which are difficult to control during the tests.

The formulations derived in this study for predicting rainfall induced soil erosion rates are highly encouraging, as they are able to estimate the erosion rates reasonably well within the range of parameters tested during this study. In addition, these equations are also relatively easy to apply in actual situations. However, the number of soil samples tested during this study might not be sufficient to carry out a complete verification of the equations. Therefore, further tests are recommended with more soil samples and also widening the range of test parameters to verify the equations and to make it applicable over a wide range of actual situations. In addition, it is also important to verify the equations against field data prior to apply for actual cases.

\section{References}

1. Aberathna, H.M.R.M., W.T.D. Nandasiri, S.T.K. Sandamalika and K.P.P. Pathirana, "Experimental Investigation of Rainfall Induced Soil Erosion", University Research Sessions - 2007, University of Peradeniya, Sri Lanka, Vol.12, Part II, pp.179-180, 2007

2. Dimoyiannis, D.G., S. Valmis, P. Vyrlas, "A Rainfall Simulation Study of Erosion of Some Calcarcous Soils", National Agricultural Research Foundation, Institute of Soil Classification and mapping, Vol. 3, pp.179. 183, 2001.

3. Herngren, L., A. Goonetilleke, R. Supum and D.Y. De Silva, "Rainfall Simulations as a Tool for Urban Water Quality Research", Environmental Engineering Science, Vol. 12, No.3, Queensland University of Technology, Brisbane, Australia, pp. 378-383, 2005.

4. Hudson, N.W., "Raindrop Size Distribution in High Intensity Storms", Rhodesian I. Agricult. Res. Vol. 1, pp.6, 1963.

5. Lal, R., "Soil Erosion Research Method", Soil and Conservation Society, pp. 75-94, 1988.

6. Morgen, R.P.C., "Soil Erosion and Conservation", Second Edition, Longman, UK, pp.1-40, 1995.

7. Van Dijk, A.I.J.M., L. A. Bruijnzeel and E.H. Eisma, "A Methodology to Study Rain Splash and Wash Processes under Natural Rainfall", Hydrological Processes, Vol.17, pp.153-167, 2003. 